\title{
Use of an improvised pneumatic anti-shock garment and a non-pneumatic anti-shock garment to control pelvic blood flow
}

\author{
Mark Hauswald • Michael R. Williamson • \\ Gillian M. Baty • Nancy L. Kerr • \\ Victoria L. Edgar-Mied
}

Received: 14 November 2009 / Accepted: 22 April 2010 / Published online: 21 July 2010

(C) Springer-Verlag London Ltd 2010

\begin{abstract}
Background Pelvic bleeding from trauma and postpartum hemorrhage is often difficult to treat successfully by emergency providers particularly in low resource environments, when hospital presentation is delayed or there is a lack of immediate surgical, anesthesia, and transfusion capabilities. Pneumatic anti-shock garments (PASG) decrease pelvic blood flow and hemorrhage. A tightly fitted neoprene non-pneumatic anti-shock garment (NASG) has been shown to decrease blood loss and improve survival rates from postpartum hemorrhage.

Aims The objective of this study was to determine whether blood flow to the pelvis is decreased by use of the NASG or by an improvised PASG.

Methods A PASG was made using three bicycle tubes, placing one tube on each leg and one on the lower abdomen/ pelvis, wrapping firmly with sheets and inflating the tubes to approximately 3.5 bar (45 psi). A Doppler ultrasound was
\end{abstract}

An abstract of this work was presented at the FIGO meeting, October 2009 in Cape Town, Union of South Africa.

\footnotetext{
M. Hauswald $(\bowtie) \cdot$ G. M. Baty

Emergency Medicine, Global Health Programs,

University of New Mexico Health Sciences Center,

Cancer Center B-44,

Albuquerque, NM 87131-0001, USA

e-mail: MHauswald@salud.unm.edu

M. R. Williamson • V. L. Edgar-Mied

Department of Radiology, University of New Mexico

Health Sciences Center,

Albuquerque, NM, USA

N. L. Kerr

Obstetrics and Gynecology, University of New Mexico

Health Sciences Center,

Albuquerque, NM, USA
}

used to measure distal aortic blood flow in 12 healthy adults at baseline and in both devices. Data were analyzed with one sample and paired $t$ tests.

Results Mean flow was $1.99 \mathrm{l} / \mathrm{min}$ at baseline. Mean flow decrease was 1.11 [95\% confidence interval (CI): 0.64 $1.57, p=0.0003$ for the difference] for the PASG and 0.65 (95\% CI: $0.03-1.26, p=0.04$ ) for the NASG. The PASG decreased blood flow more than the NASG (mean difference: $0.46,95 \%$ CI: $0.02-0.90, p=0.04$ ).

Conclusions Both devices decreased distal aortic blood flow, but the improvised PASG device decreased it by a larger margin.

Keywords Pelvic trauma - Emergency obstetric care . Postpartum hemorrhage $\cdot$ Anti-shock trousers

\section{Introduction}

Hemorrhage from pelvic blood vessels after trauma or obstetric delivery are major health care problems. Trauma is a leading cause of death worldwide and a major cause of lost quality-adjusted life years (QALYs) [1]. Treatment of pelvic trauma is difficult since hemorrhage can be massive and it is difficult to apply direct pressure to the bleeding vessels. Postpartum hemorrhage is the leading cause of obstetric deaths and causes an estimated 150,000 maternal deaths per year [1]. Definitive control of severe pelvic bleeding from any cause often requires aggressive measures such as arterial embolization, laparotomy, internal fixation, or pelvic packing. These procedures are difficult to perform because they require immediate access to sophisticated medical and surgical expertise and equipment. Severe hemorrhage is often aggravated by disseminated intravas- 
cular coagulation. Blood volume must be maintained by transfusion of red blood cells, platelets, and clotting factors. Transfusion requirements can be massive and overwhelm the capacity of even large facilities. These issues are particularly difficult in low resource environments.

Ideally pelvic hemorrhage could be controlled by simple external pressure. This could provide time to mobilize more sophisticated resources and might decrease transfusion requirements and the need for aggressive therapy. Pneumatic anti-shock garments (PASG), also called medical or military anti-shock trousers (MAST), have been shown to greatly decrease pelvic blood flow [2, 3], and case reports imply that they are effective in patients with pelvic injuries [4] and postpartum hemorrhage [5]. PASG have little or no benefit in treating trauma patients in general [6]. However, most of the patients enrolled in clinical trials had thoracic or abdominal trauma and had ready access to definitive surgery. In addition PASG have little effect on the general circulation. They only autotransfuse about $250 \mathrm{cc}$ of blood [7] from the lower body into the central circulation and have little or no effect on cardiac output [2]. These findings would explain differing outcomes for upper body versus pelvic hemorrhage. In their favor PASG have been used thousands of times and have proven quite safe. Unfortunately PASG are complicated, expensive, and rarely available in the low resource areas where they would seem most useful. A simple and less expensive alternative to PASG was developed by The National Aeronautics and Space Administration (NASA). This non-pneumatic antishock garment (NASG) is made of neoprene and is tightly applied to the patient using Velcro $^{\mathrm{TM}}$ straps. It has been commercialized as the ZOEX Non-Inflatable Anti-Shock Garment $^{\mathrm{TM}}$ (ZOEX, Ashland, OR, USA). The NASG has been shown to decrease postpartum blood loss by $50 \%[8$, 9] and improve survival rates from postpartum hemorrhage [10]. Although the NASG is less expensive than commercial PASGs it currently has limited availability.

We designed a PASG that can be made from inexpensive and easily available supplies. We measured pelvic blood flow in volunteers before and after being placed in the NASG and PASG. Our null hypothesis was that neither device would affect blood flow to the pelvis.

\section{Materials and methods}

Review and approval of the study protocol was obtained from our Institutional Review Board. Twelve healthy nonpregnant adult volunteers were selected. Subjects ranged in age from 21 to 60 years of age and weighed $52-82 \mathrm{~kg}$. The sample size was based on previous research [3]. Written informed consent was obtained from each subject. A physician was present during each trial.
An Acuson Sequoia 512 Ultrasound and 4VI probe operating at 2-3 MHz (Siemens, Mountain View, CA, USA) was used to measure heart rate, apparent artery diameter, Doppler-to-vessel angle, and blood flow velocity at the abdominal aorta below the superior mesenteric artery (SMA). These were used to calculate flow rate (volume per time). Details of this technique have been published elsewhere [3]. Measurements were obtained from each subject at baseline and immediately after being placed in each device. Device order was alternated between subjects with the initial subject's order mechanically randomized. The PASG was made by wrapping individual folded hospital sheets around each leg and the abdomen/pelvis. After the first wrap a bicycle tube was placed on each sheet and the sheet was further wrapped around the patient and tube. A second set of three sheets was then wrapped firmly around the first set being careful to allow the tube stem but no other part to protrude. The tubes were then inflated with a bicycle pump to $2.8-3.5$ bar (40-50 psi). The ultrasonographer has 20 years experience and is certified by the American Registry for Diagnostic Medical Sonography (Rockville, MD, USA) as both a registered diagnostic medical sonographer (RDMS) and a registered vascular technician (RVT). Data were analyzed with one sample and paired $t$ tests using two-tailed $p$ values.

\section{Results}

Mean blood flow was $1.99 \mathrm{l} / \mathrm{min}$ at baseline with a range of $0.39-3.05 \mathrm{l} / \mathrm{min}$. Mean flow decrease was $1.11 \mathrm{l} / \mathrm{min}$ or $56 \%$ [95\% confidence interval (CI): $0.64-1.57, p=0.0003$ for the difference] for the PASG and $0.651 / \mathrm{min}$ or $33 \%$ (95\% CI: $0.03-1.26, p=0.04$ ) for the NASG. The PASG decreased blood flow more than the NASG (mean difference: $0.46,95 \%$ CI: $0.02-0.90, p=0.04$ ).

\section{Discussion}

Our results are compatible with the medical literature regarding the regional hemodynamic effects of circumferential abdominal pelvic pressure using PASG [2, 3]. Commercial PASG are quite rigid and their air bladders are considerably larger than bicycle tubes. A previous study showed that when inflated to two thirds of their maximum, commercial PASG decrease blood flow by approximately one half, which is similar to the magnitude of decrease we demonstrated with our improvised PASG. Fully inflated commercial PASG decrease distal aortic flow by about three quarters $[2,3]$.

Pelvic hemorrhage and particularly postpartum hemorrhage is multifactorial. Bleeding can be arterial from 
branches of the internal iliac, pelvic, ovarian, and middle sacral arteries. Bleeding can also be from veins, capillaries, and uterine spiral arterioles. Bleeding will stop when externally applied pressure exceeds the perfusion pressure of the injured vessel. Low pressure venous bleeding theoretically requires only a few millibars, but this must be at the actual bleeding sites. This can be difficult to apply when the affected vessels are pelvic or retroperitoneal. Complete elimination of arterial bleeding requires pressure that exceeds the systemic blood pressure. This is impossible to apply externally and would in addition completely eliminate tissue perfusion, causing undesirable side effects.

Circumferential pressure can be viewed as having two independent effects on hemorrhage. It provides indirect pressure on the pelvic organs that exceeds venous and capillary filling pressure. This stops bleeding from these sources. Circumferential pressure also decreases actual arterial flow. This directly decreases arterial bleeding and hence should give normal hemostatic mechanisms more opportunity to act. It is unfortunate that the term "antishock" became incorporated into the names of these devices because they have essentially no utility in reversing shock in general or in preventing it from injuries that are above the pelvis. However, emergency care and childbirth providers need a means of slowing or stopping pelvic hemorrhage. This is particularly true in low resource environments or when patients present late or to facilities that lack interventional radiology, immediate surgical capability, and safe blood. Delays in gaining access to definitive care are even more serious if patients suffer from preexisting anemia or have little reserve.

Although we did not do any formal testing of device acceptability, we did ask all of the subjects if they wanted the trial terminated because the devices were too uncomfortable and none did. One subject had participated in a previous PASG study and spontaneously volunteered that all three devices felt "about the same" which is reassuring given the long clinical experience with commercial PASG.

Limitations of our study include the use of nonpregnant subjects. The postpartum uterus is larger and has greater blood flow than the nonpregnant uterus. How shock affects perfusion of the postpartum uterus is not known. In addition our subjects were euvolemic and not bleeding. Hypovolemia ordinarily causes peripheral vasoconstriction which would lead to less blood flow at the distal aorta. However, sepsis and late shock cause vasodilatation which causes opposite changes. It would be unethical and impractical to perform an initial trial on ill and bleeding subjects. Our within-subject experimental design specifically compensates for differing amounts of pelvic blood flow between subjects. It should also compensate for the above physiologic states. Although the amount of blood flow in the distal aorta might be different, the effect of mechanical pressure and hence the comparison between devices would be the same. Although the improvised device can be made locally, the NASG is available in a manufactured version which may make it more suitable in places that can afford the initial investment. The PASG decreased blood flow by a larger margin than the NASG, but this does not prove that it is clinically either equivalent or superior. It should be noted that the optimal pressure to treat pelvic and postpartum bleeding is not known but that the pressure in each device can be changed by adding or removing air from the tubes or adjusting the tension on the straps of the NASG. Currently available reports of NASG use in obstetric hemorrhage have not noted problems with compartment syndrome, but the number of subjects studied is small. Randomized controlled clinical trials of both devices are needed.

Acknowledgements The ZOEX Non-Inflatable Anti-Shock Garment $^{\mathrm{TM}}$ was supplied by ZOEX, P.O. Box 435, Ashland, OR 97520, USA.

Conflict of interest None.

\section{References}

1. World Health Organization (2009) Cause-specific mortality and morbidity. http://www.who.int/whosis/whostat/EN_whs09_table2. pdf. Accessed 13 Nov 2009

2. Hauswald M, Greene ER (1986) Aortic blood flow during sequential MAST inflation. Ann Emerg Med 15:1297-1299

3. Hauswald M, Greene ER (2003) Regional blood flow after pneumatic anti-shock garment inflation. Prehosp Emerg Care 7:225-228

4. Clarke G, Mardel S (1993) Use of MAST to control massive bleeding from pelvic injuries. Injury 24:628-629

5. Moyes DG (1985) The MAST suit in the treatment of severe postpartum hemorrhage. S Afr Med J 67:157

6. Mattox KL, Bickell W, Pepe PE, Burch J, Feliciano D (1989) Prospective MAST study in 911 patients. J Trauma 29:11041111

7. Hanke BK, Bivins HG, Knopp R, dos Santos PA (1985) Antishock trousers: a comparison of inflation techniques and inflation pressures. Ann Emerg Med 14:636-640

8. Hensleigh PA (2002) Anti-shock garment provides resuscitation and haemostasis for obstetric haemorrhage. BJOG 109:13771384

9. Miller S, Turan JM, Dau K, Fathalla M, Mourad M, Sutherland T et al (2007) Use of the non-pneumatic anti-shock garment (NASG) to reduce blood loss and time to recovery from shock for women with obstetric haemorrhage in Egypt. Glob Public Health 2:110-124

10. Miller S, Ojengbede O, Turan JM, Morhason-Bello IO, Martin HB, Nsima D (2009) A comparative study of the non-pneumatic anti-shock garment for the treatment of obstetric hemorrhage in Nigeria. Int J Gynaecol Obstet 107:121-125 